\section{Schützt Gruppenintervention ältere Pflegekräfte vor dem Burnout?}

\begin{abstract}
Ältere Pflegende werden häufig durch die hohen psychischen und physischen Anforderungen ihres Berufes selbst krank und geben ihren Beruf vorzeitig auf. Eine gezielte psychologischen Gruppenintervention bei Pflegern über 45 Jahre kann die gesundheitsbezogene Lebensqualität und ihre emotionale Belastbarkeit erhöhen, wie eine deutsche Studie zeigt.
\end{abstract}

A n der aktuellen multizentrischen, randomisierten, kontrollierten Interventionsstudie von Maatouk et. al. an vier deutschen Kliniken (Heidelberg, Ulm, Aalen und Düsseldorf) nahmen 115 Pflegekräfte teil. Die eine Hälfte der Pflegenden erhielt sechs zweistündige Präventionsseminare in kleinen Gruppen von jeweils zehn Teilnehmern im Abstand von einer Woche. Die Intervention wurde mit einer Auffrischungssitzung nach sechs Wochen beendet. Die andere Hälfte wurde auf eine Warteliste gesetzt und diente als Vergleichsgruppe. Die Probanden waren im Schnitt 52 Jahre alt und hatten bereits 30 Berufsjahre hinter sich (47 Frauen und sieben Männer, davon arbeiteten 17 in Voll- und 37 in Teilzeit).

$37 \%$ von ihnen pflegten zu Hause zusätzlich Angehörige. Im Quartal vor der Befragung hatte jeder der Studienteilnehmer durchschnittlich 3,15 Fehltage. In der ersten Sitzung wurden die Schwierigkeiten, die mit zunehmenden Alter immer mehr auftreten, mögliche Res- sourcen sowie individuelle Schutzfaktoren diskutiert. Die zweite Sitzung widmete sich der Arbeitsbiographie, reflektierte die Ressourcen und die Erfahrungen, wie Schwierigkeiten bislang bewältigt wurden. In der dritten Sitzung lag der Fokus auf der Stressbewältigung, das problem- und gefühlszentrierte Coping und die Entspannungstechniken.

Die darauf folgenden drei Runden legten den Fokus auf das SOC-Manual (Selektion, Optimierung und Compensation): Es galt wenige, aber dafür gut überlegte Ziele zu formulieren und diese optimal umzusetzen sowie Hindernisse durch kompensatorische Mittel zu bewältigen. Die sechste Sitzung widmete sich den Vor- und Nachteilen des höheren Alters im Beruf und reflektierte Stereotypien.

Der Erfolg der Prävention wurde mit den WHO-Selbstbewertungsfragebögen zum Wohlbefinden und der psychologischen mentalen Lebensqualität vor und nach der Intervention evaluiert. Ein sekundäres Studienziel war die Bewertung der kognitiven und emotionalen Irritierbarkeit. Zu diesem Projekt wurden alle gesunden Pfleger der teilnehmenden Kliniken über 45 Jahre eingeladen. Die Mitarbeit fand während der Arbeitszeit statt. Von den 54 Teilnehmern der Interventionsgruppe beendeten 51 den Kurs.

\section{Bessere mentale Lebensqualität und emotionale Belastbarkeit}

Die Intervention verbesserte die mentale gesundheitsbezogene Lebensqualität. Signifikant nahmen auch die Werte für die Irritation $a b$, einem Parameter für die emotionale und kognitive Beanspruchung durch den Beruf. Die Teilnehmer gaben am Ende der Studie beispielsweise an, nach der Arbeit besser abschalten zu können und weniger müde oder gereizt zu sein.

Länger angelegte Studien zur SOCPrävention könnten außerdem zeigen, ob das vorzeitige Ausscheiden aus dem Beruf sich damit vermeiden ließe. Die Therapiekosten von 436 Euro/Teilnehmer jedenfalls sind im Vergleich zum Schaden durch lange Krankschreibungen oder dem frühen Berufsausstieg relativ gering. Stephanie Mühlbauer

I.Maatouk et. al.: Healthy ageing at work. Efficacy of group interventions on the mental health of nurses aged 45 and older: Results of a randomised, controlled trial. Plos One, Januar 2018. http://journals.plos.org/plosone/article?10.1371/journal.pone.0191000

\title{
Keimschleuder Pflegekittel
}

\section{Bis zu 30\% der Pflegekittel sind nach US-Daten mit pathogenen Keimen kontaminiert. Der Grad der Verunreinigung hängt dabei von der Tätigkeit der Pflegekräfte ab.}

\footnotetext{
nfekte mit Krankenhauskeimen sind ein großes Problem. Auch das Pflegepersonal kann Keime auf der Station verbreiten - etwa über kontaminierte Arbeitskleidung. Wie häufig die Kleidung des Pflegepersonals mit pathogenen Keimen belastet ist und welche Tätigkeiten die Belastung besonders erhöhen, hat ein Team um Dr. Kerri Thom von der Maryland School of Medicine in
}

Baltimore eruiert. Zu diesem Zweck verteilten die Epidemiologen neue Kittel an insgesamt 90 Pflegekräfte auf Intensivund Wachstationen von Kliniken der Universität von Maryland. Jeder Teilnehmer erhielt im Laufe von acht Monaten vier neue Kittel, von diesen wurden achtmal unangemeldet Proben in den letzten vier Stunden einer Schicht genommen.
Dazu wurde mit einem sterilen feuchten Tuch großflächig über den Kittel gewischt und zusätzlich eine Agarplatte direkt auf den Bauchbereich des Kleidungstücks gedrückt. Die Bakterien wurden anschließend auf gramnegative Erreger, Staphylokokken sowie Enterokokken hin untersucht.

\section{Die Kontamination war abhängig von der Tätigkeit}

Die Träger hatten im Schnitt an 73\% der Probenentnahmetage Patienten mit Wunden behandelt, an 70\% solche mit maschineller Beatmung, an 68\% Patienten mit besonderen Kontakt-Vorsichtsmaßnahmen und an 52\% solche mit 
Durchfall. Gebadet hatten sie Patienten an $29 \%$ der Tage. Insgesamt wurden 720 Proben genommen. 30\% wiesen pathogene Keime auf. In jeweils 16\% aller Proben fanden die Studienautoren S. aureus und gramnegative Erreger. Enterokokken ließen sich bei 3\%, multiresistente Erreger bei 6\% nachweisen. 33\% der Proben waren von Trägern kontaminiert, die Patienten mit Wunden versorgt, und $36 \%$ von solchen, die Patienten gebadet hatten. Nach Durchfallbehandlungen ... llpatienten war die Rate etwas erhöht (33\%).

Unter Berücksichtigung einer Reihe von Begleitfaktoren fanden die Forscher um Thom ein signifikant erhöhtes Risiko für Kontaminationen nach einer
Wundbehandlung (Odds Ratio, OR = 1,75). Berücksichtigten sie auch den Kontaminationsgrad - also die Zahl der Kolonien-bildenden Einheiten - ergab sich eine knapp signifikant stärkere Belastung bei Trägern nach einem Patientenbad. Bei Pflegekräften mit einem Kittel, der mehr als 16 Tage alt war, zeigte sich ein Trend zu einer stärkeren Belastung. Dagegen waren die Kittel signifikant weniger stark kontaminiert, wenn die Träger Patienten mit besonderen Kontaktvorsichtsmaßnahmen behandelt hatten.

(tm)

Thom KA et al. Frequent contamination of nursing scrubs is associated with specific care activities. American Journal of Infection Control 2018; online 11. Jan. 2018

\section{Herzattacken durch Wetterwechsel}

Eben war es noch ganz kalt, und plötzlich ist es 20 oder 25 Grad wärmer. Die zunehmenden Wetterkapriolen setzen Herzpatienten besonders zu. Wissenschaftler der Universität Michigan konnten bei 30.000 Patienten, die über sechs Jahre beobachtet wurden, zeigen, dass Temperaturerhöhungen das Risiko für Herzattacken erhöhen. Ein Temperaturanstieg um 5 Grad Celsius steigert das Infarktrisko um 5\%, ein plötzlicher Anstieg um 25\% lässt das Risiko entsprechend deutlicher hochschnellen. An Tagen mit extremen Temperaturschwankungen treten fast doppelt so viele Herzinfarkte auf als an Tagen mit relativ konstanten Temperaturen. Gefährlich wird es besonders an warmen Tagen. Eine Kausalität ist noch nicht belegt. Es kann jedoch nicht schaden, wackelige Herzpatienten an Tagen mit Wetterka-priolen zu nur vorsichtigen Aktivitäten zu raten.

ACC-Kongress 2018

\title{
Fachkräftemangel verursacht Hygienedefizite
}

ANZEIGE

\author{
Trotz einiger Erfolge bei der Verbesserung der Kran- \\ kenhaushygiene und im Kampf gegen MRSA weist \\ Deutschland nach wie vor erhebliche Schwächen auf.
}

$\mathrm{D}$ eutschland steht bei der Verbesserung der Krankenhaushygiene und im Kampf gegen multiresistente Keime weiter vor erheblichen Herausforderungen. Diese Bilanz zog der Präsident der Deutschen Gesellschaft für Krankenhaushygiene, Professor Martin Exner, anlässlich des 14. Kongresses für Krankenhaushygiene im März in Berlin. Die Herausforderung sei die zunehmende Alterung mit mehr multimorbiden Patienten, die ein erhöhtes Risiko für schwere Infektionen haben. So sollte auch die Vermittlung einer hygienischen Grundkompetenz bei pflegenden Angehörigen eine Standard-Pflichtleistung der Pflegeversicherung werden. Ein besonderes Problem in diesem Jahr sei die Influenza-Epidemie gewesen, die auch das Pflegepersonal betroffen und zu Versorgungsengpässen geführt habe. „Die Motivation zum Impfen ist dringend verbesserungsbedürftig", mahnte Exner.

Als inakzeptabel bewertet die Fachgesellschaft die Ausstattung deutscher Kliniken mit Pflegepersonal: Mit einer Relation von 13 Patienten auf eine Fachpflegekraft rangiere Deutschland in Europa auf dem untersten Platz, noch hinter Griechenland. Erstrebenswert sei eine Relation von eins zu sieben, was dem niederländischen Niveau entspricht. Die Unterbesetzung in der Pflege verursache eine zunehmende Verdichtung von Arbeitsabläufen, die auch zu Infektionsrisiken führe. Entsprechende Forderungen des Deutschen Pflegerats würden ausdrücklich unterstützt.



14. Kongress für Krankenhaushygiene, 18. -21. März 2018, Berlin. 